\title{
SOLVING THE GLEASON PROBLEM ON LINEARLY CONVEX DOMAINS
}

\author{
OSCAR LEMMERS AND JAN WIEGERINCK
}

\begin{abstract}
Let $\Omega$ be a bounded, connected linearly convex set in $\mathbb{C}^{n}$ with $C^{1+\epsilon}$ - boundary. We show that the maximal ideal (both in $A(\Omega)$ and $H^{\infty}(\Omega)$ ) consisting of all functions vanishing at $p \in \Omega$ is generated by the coordinate functions $z_{1}-p_{1}, \ldots, z_{n}-p_{n}$.
\end{abstract}

\section{INTRODUCTION}

Let $\Omega$ be a bounded domain in $\mathbb{C}^{n}$. Let $R(\Omega)$ (usually $A(\Omega)$ or $H^{\infty}(\Omega)$ ) be a ring of holomorphic functions that contains the polynomials, and let $p=\left(p_{1}, \ldots, p_{n}\right)$ be a point in $\Omega$. Recall the Gleason problem, cf. [8]: is the maximal ideal in $R(\Omega)$ consisting of functions vanishing at $p$, generated by the coordinate functions $\left(z_{1}-p_{1}\right)$, $\ldots,\left(z_{n}-p_{n}\right) ?$

One says that a domain $\Omega$ has the Gleason R-property if this is the case for all points $p \in \Omega$. We also say that it has the Gleason-property with respect to $R(\Omega)$. Leibenzon was the first to solve a non trivial Gleason problem. He proved ([1]) that the Gleason problem can be solved on any convex domain in $\mathbb{C}^{n}$ having a $C^{2}$ boundary. This result was sharpened by Grangé ([9], for $H^{\infty}(\Omega)$ ), and by Backlund and Fällström ([3] and [], for $H^{\infty}(\Omega)$ and $A(\Omega)$ respectively), for convex domains in $\mathbb{C}^{n}$ having only a $C^{1+\epsilon}$-boundary.

Using his theorem on solvability of the $\bar{\partial}$-problem ([14]), Øvrelid proved in [15] that a strictly pseudoconvex domain in $\mathbb{C}^{n}$ with $C^{2}$-boundary has the Gleason $A$ property. Fornæss and Øvrelid showed in 7 that a pseudoconvex domain in $\mathbb{C}^{2}$ with real analytic boundary has the Gleason $A$-property. This was extended by Noell ([13]) to pseudoconvex domains in $\mathbb{C}^{2}$ having a boundary of finite type.

Backlund and Fällström proved in [6] that a bounded, pseudoconvex Reinhardt domain in $\mathbb{C}^{2}$ with $C^{2}$-boundary that contains the origin, has the Gleason $A$-property. The present authors showed in 12 that one does not need that $\Omega$ is pseudoconvex or that it contains the origin. They also solved the $H^{\infty}$-problem for such Reinhardt domains.

Note that there are not always solutions to the Gleason problem; in fact, Backlund and Fällström showed $(\sqrt[5]{5})$ that there even exists an $H^{\infty}$-domain of holomorphy on which the problem is not solvable.

In this article, we return to the original method of Leibenzon, and use it to solve the Gleason problem on $\mathbb{C}$-convex domains (these are domains such that their intersection with any complex line passing through the domain is connected and simply connected) in $\mathbb{C}^{n}$ with $C^{1+\epsilon}$-boundary. We denote the derivate of a function $g$ with

Date: June 27, 2001.

1991 Mathematics Subject Classification. Primary : 32A38; Secondary : 32F17.

Key words and phrases. Gleason problem, linearly convex set, $\mathbb{C}$-convex set. 
respect to the $k$ 'th coordinate with $D_{k} g$. After translation we can assume that the domain contains the origin, and that $p=0$. If $\Omega$ is convex, it is easy to see that for $f_{i}(z):=\int_{0}^{1} D_{i} f(\lambda z) d \lambda, f(z)=\sum_{i=1}^{n} z_{i} f_{i}(z)$. The hard part is to show that $f_{i} \in A(\Omega)$. Leibenzon did this by making estimates of $D_{i} f(\lambda z)$ on the line segment between 0 and 1 . If one considers $\mathbb{C}$-convex domains, this method doesn't work, of course. However, for a polynomial $P$, and

$$
T_{i}(P)(z):=\int_{0}^{1} D_{i} P(\lambda z) d \lambda
$$

we still have that $P(z)=\sum_{i=1}^{n} z_{i} T_{i}(P)(z)$. The theorem of Cauchy gives

$$
T_{i}(P)(z)=\int_{0}^{1} D_{i} P(\lambda z) d \lambda=\int_{\gamma_{z}} D_{i} P(\lambda z) d \lambda
$$

for any curve $\gamma_{z}$ in $\mathbb{C}$ that connects 0 and 1 . We choose this curve $\gamma_{z}$ such that for all $s \in[0,1]$ the point $\gamma_{z}(s) z$ is in $\bar{\Omega}$ intersected with the complex line through 0 and $z$. Estimating $D_{i} P(\lambda z)$ on $\gamma_{z}$ yields an estimate of $T_{i}(P)(z)$ in terms of $\|P\|_{S}$ (where $S$ is a suitable compact subset of $\Omega$ ) instead of $\|P\|_{\tilde{\Omega}}$ (where $\tilde{\Omega}$ is the convex hull of $\Omega$ ). Then the fact that $\Omega$ is a Runge domain is used, first to extend $T_{i}$ to $H^{\infty}(\Omega)$, then to $A(\Omega)$.

\section{2. $\mathbb{C}$-CONVEX SETS}

In $\mathbb{R}^{n}$ there are two natural definitions of convexity. A set $E$ is convex if

1. The intersection of $E$ with each line is connected, or

2. Through every point in the complement of $E$ there passes a hyperplane which does not intersect $E$.

If one assumes that $E$ is connected, these definitions are equivalent. These definitions lead to the following generalizations of convexity in $\mathbb{C}^{n}$ :

1. One says that a set $\Omega \subset \mathbb{C}^{n}$ is $\mathbb{C}$-convex if all its intersections with complex lines are connected and simply connected.

2. $\Omega$ is said to be linearly convex (also : lineally convex) if through every point in the complement of $\Omega$ there passes a complex hyperplane that does not intersect $\Omega$.

3. An open set $\Omega$ in $\mathbb{C}^{n}$ is called weakly linearly convex if through every point of $\partial \Omega$ there passes a complex hyperplane that does not intersect $\Omega$.

For proofs of the following assertions and more information on $\mathbb{C}$-convex sets we refer to [1], [2] and [10.

- Every $\mathbb{C}$-convex set is pseudoconvex.

- Every $\mathbb{C}$-convex set is a Runge domain.

- For a bounded connected domain $\Omega$ with $C^{1+\epsilon}$-boundary all the previous definitions are equivalent, and every complex line passing through $\Omega$ will intersect $\partial \Omega$ transversally.

\section{Definitions and auxiliary Results}

We establish some notations : by $\|f\|_{\Omega}$ we denote the supremum of $|f|$ on $\Omega$. We denote the derivate of a function $g$ with respect to the $k$ 'th coordinate with $D_{k} g$. The unique complex line through the points 0 and $z$ is denoted by $L_{0, z}$. For a $w \in \partial \Omega$, we denote by $n_{w}$ the inner unit normal vector to $\partial \Omega$ at $w$. Given $z \in \mathbb{C}^{n}$, we denote by $\pi_{z}\left(n_{w}\right)=\left(\pi_{z}\left(n_{w}\right)_{1}, \ldots, \pi_{z}\left(n_{w}\right)_{n}\right)$ the orthogonal projection of $n_{w}$ 
onto $L_{0, z}$. Constants may be denoted by the same letter at various places, even though their values are different.

Lemma 1. (Cf. 团.) Let $w \in \partial \Omega$. There exist a neighborhood $W$ of $w$ and a $\sigma>0$ such that for all $z \in W \cap \partial \Omega$, and e a complex unit tangent vector to $\partial \Omega$ at $z$, the following holds : if $0<s<1$ then $|t|<(1-s)^{1 /(1+\epsilon / 2)}<\sigma$ implies that $z+(1-s) \pi_{z}\left(n_{w}\right)+t e \in \Omega$.

Proof. Since $\Omega$ has $C^{1+\epsilon}$-boundary, there is a $C^{1+\epsilon}$-defining function $r$ with $\nabla r \neq 0$ on $\partial \Omega$, such that $\Omega=\left\{z \in \mathbb{C}^{n}: r(z)<0\right\}$. We compute at $z \in W \cap \Omega$ :

$$
\begin{aligned}
& r\left(z+(1-s) \pi_{z}\left(n_{w}\right)+t e\right)-r(z) \\
& =\Re\left(\sum_{j=1}^{n} \frac{\partial r}{\partial z_{j}}(z)\left((1-s) \pi_{z}\left(n_{w}\right)_{j}+t e_{j}\right)\right)+O\left(\left|(1-s) \pi_{z}\left(n_{w}\right)+t e\right|^{1+\epsilon}\right) \\
& =(1-s) \Re\left(\sum_{j=1}^{n} \frac{\partial r}{\partial z_{j}}(z) \pi_{z}\left(n_{w}\right)_{j}\right)+(1-s) g(s, t),
\end{aligned}
$$

with

$$
g(s, t)=O\left(\left|\frac{s-1}{(1-s)^{1 /(1+\epsilon)}} \pi_{z}\left(n_{w}\right)+\frac{t e}{(1-s)^{1 /(1+\epsilon)}}\right|^{1+\epsilon}\right),
$$

since $e$ is a complex tangent vector to $\partial \Omega$ at $z$, causing $\Re\left(\sum_{j=1}^{n} \frac{\partial r}{\partial z_{j}}(z) t e_{j}\right)$ to vanish. Let $1-s, t$ tend to zero with $|t|<(1-s)^{1 /(1+\epsilon / 2)}$. Then the term

$$
\frac{s-1}{(1-s)^{1 /(1+\epsilon)}} \pi_{z}\left(n_{w}\right)+\frac{t e}{(1-s)^{1 /(1+\epsilon)}}
$$

tends to zero. For every $1-s$ close to zero, there is a small $t$ such that $z+(1-$ $s) \pi_{z}\left(n_{w}\right)+t e \in \Omega$, in other words : $r\left(z+(1-s) \pi_{z}\left(n_{w}\right)+t e\right)<0$. Since $\pi_{z}\left(n_{w}\right)$ is not a complex tangent vector (if we choose $W$ small enough), $\Re\left(\sum_{j=1}^{n} \frac{\partial r}{\partial z_{j}}(z)(1-\right.$ $\left.s) \pi_{z}\left(n_{w}\right)_{j}\right) \neq 0$. Hence this term has to be negative. Then there is a constant $\sigma>0$ such that $|t|<(1-s)^{1 /(1+\epsilon / 2)}<\sigma$ implies that

$$
(1-s) \Re\left(\sum_{j=1}^{n} \frac{\partial r}{\partial z_{j}}(z) \pi_{z}\left(n_{w}\right)_{j}\right)+(1-s) g(s, t)<0,
$$

in other words : $z+(1-s) \pi_{z}\left(n_{w}\right)+t e \in \Omega$.

Lemma 2. For every $z \in \partial \Omega$ there exists a neighborhood $W$ of $z$ and $a \quad \sigma>0$ such that $F_{z}(w, s):=w+(1-s) \pi_{w}\left(n_{z}\right)$ is a bijection from $W \cap \partial \Omega \times(1-\sigma, 1]$ to $W \cap \bar{\Omega}$.

Proof. Observe that $\pi_{z}\left(n_{z}\right) \neq 0$ (since $L_{0, z}$ intersects $\partial \Omega$ transversally), such that the Jacobian of $F_{z}(w, s)$ does not vanish at $(z, 1)$. Now apply the inverse function theorem.

Compactness of $\partial \Omega$ and lemma 1 and 2 now yield :

Corollary 1. There exist a finite number of open sets $W_{1}, \ldots, W_{m}$ in $\mathbb{C}^{n}$ containing points $w_{1}, \ldots, w_{m}$ respectively, and a $\sigma>0$ such that :

- $\partial \Omega \subset \cup W_{i}$

- $F_{w_{i}}(w, s): W_{i} \cap \partial \Omega \times(1-\sigma, 1] \rightarrow W_{i} \cap \bar{\Omega}$ is a bijection for all $1 \leq i \leq m$. 
- For $w \in W_{i}$, and e a complex unit tangent vector to $\partial \Omega$ at $w$ : if $0<s<1$ and $|t|<(1-s)^{1 /(1+\epsilon / 2)}<\sigma$, then $w+(1-s) \pi_{w}\left(n_{w_{i}}\right)+$ te $\in \Omega \quad \forall 1 \leq i \leq m$.

Let $V_{i}:=\left\{z \in \bar{\Omega}: \exists w \in W_{i}, s \in\left(1-\frac{1}{2} \sigma, 1\right]: z=w+(1-s) \pi_{w}\left(n_{w_{i}}\right)\right\}, V:=\cup_{i} V_{i}$. Since $\Omega \backslash V$ is compact in $\Omega$, we have that $A:=\min d(\partial \Omega, \Omega \backslash V)>0$.

Definition. For $z \in V$ we say that $w, w_{k} \in W_{k}$ correspond to $z$ if there is an $s \in\left(1-\frac{1}{2} \sigma, 1\right]$ such that $z=w+(1-s) \pi_{w}\left(n_{w_{k}}\right)$.

For $z \notin V$ we fix a smooth curve $\gamma_{z}$ in $\mathbb{C}$, from 0 to 1 , without loops, such that

$$
\gamma_{z}(s) z \in \Omega \text { and } \quad d\left(\gamma_{z}(s) z, \partial \Omega\right) \geq A \quad \forall s \in[0,1] .
$$

For $z \in V$ with corresponding $w$ and $w_{k}$, we fix a smooth curve $\gamma_{z}$ in $\mathbb{C}$, from 0 to 1 , without loops, that consists of two parts $\gamma_{z}^{1}$ and $\gamma_{z}^{2}$. We choose $\gamma_{z}^{2}$ such that for $s \in\left[1-\frac{1}{2} \sigma, 1\right], \gamma_{z}^{2}(s) z=z+(1-s) \pi_{w}\left(n_{w_{k}}\right)$, thus, with $\mu_{z}$ the unique constant such that $\mu_{z} z=\pi_{w}\left(n_{w_{k}}\right): \gamma_{z}^{2}(s)=1+(1-s) \mu_{z}$ for all $s \in\left[1-\frac{1}{2} \sigma, 1\right]$. Note that for $z \in V_{k}, \mu_{z}$ is bounded.

We choose the curve $\gamma_{z}^{1}$ in $\mathbb{C}$, from 0 to $1-\frac{1}{2} \sigma$, without loops, such that

$$
\gamma_{z}^{1}(s) z \in \Omega \text { and } d\left(\gamma_{z}^{1}(s) z, \partial \Omega\right) \geq A \quad \forall s \in\left[0,1-\frac{1}{2} \sigma\right] .
$$

It is possible to choose the curves $\gamma_{z}$ such that there is a constant $M$ with $\left|\gamma_{z}^{\prime}(s)\right| \leq$ $M \forall z, s$, and that $z_{n} \rightarrow z$ implies that $\gamma_{z_{n}}^{\prime}(s) \rightarrow \gamma_{z}^{\prime}(s) \forall z, s$.

Definition. For a polynomial $P$ that vanishes at 0 we define linear operators $T_{i}$ $(1 \leq i \leq n)$ as follows :

$$
T_{i}(P)(z):=\int_{0}^{1} D_{i} P(\lambda z) d \lambda .
$$

The operators are clearly related to the differentiated simplex functionals $\mu_{a, b}(\partial f)=$ $\int_{0}^{1} \partial f(a+t(b-a)) d t$, that are studied in [2], 3.2. Note that one has

$$
P(z)=\int_{0}^{1} \frac{d P(\lambda z)}{d \lambda} d \lambda=\int_{0}^{1} \sum_{i=1}^{n} z_{i} D_{i} P(\lambda z) d \lambda=\sum_{i=1}^{n} z_{i} T_{i}(P)(z) .
$$

\section{The Key ESTIMATE}

Lemma 3. Let $P$ be a polynomial that vanishes at 0 . Let $p \in \Omega$. There exist a constant $K$ that depends only on $\Omega$, a neighborhood $B$ of $p$ and a compact set $S$ in $\Omega$, such that $\left\|T_{i}(P)\right\|_{B} \leq K\|P\|_{S}$.

Proof. First we consider the case that $p \notin V$. Then we choose $B \subset \Omega$ such that $\bar{B} \cap V=\emptyset$. Let $z \in \bar{B}$. Then $d\left(\gamma_{z}(s) z, \partial \Omega\right) \geq A$ for all $s \in[0,1]$. If $a_{i}$ is the $i$ 'th unit vector, we have

$$
\begin{aligned}
T_{i}(P)(z) & =\int_{0}^{1} D_{i} P(\lambda z) d \lambda=\int_{\gamma_{z}} D_{i} P(\lambda z) d \lambda \\
& =\left.\int_{\gamma_{z}} \frac{d P\left(\lambda z+t a_{i}\right)}{d t}\right|_{t=0} d \lambda=\left.\int_{0}^{1} \frac{d P\left(\gamma(s) z+t a_{i}\right)}{d t}\right|_{t=0} \gamma^{\prime}(s) d s .
\end{aligned}
$$

We construct a compact $S$ such that for all $s \in[0,1] S$ contains the circle in the complex line through $s z$ and $s z+a_{i}$ with center $s z$ and radius $A$. Then we have 
for all $s \in[0,1]$ :

$$
\left|\frac{d P\left(\gamma(s) z+t a_{i}\right)}{d t}\right|_{t=0}|=| \frac{1}{2 \pi i} \int_{C(0, A)} \frac{P\left(\gamma(s) z+t a_{i}\right)}{t^{2}} d t \mid \leq \frac{\|P\|_{S}}{A},
$$

where $C(0, A)$ is the circle with center 0 and radius $A$. Hence

$$
\left|\int_{\gamma_{z}} \frac{d P\left(\lambda z+t a_{i}\right)}{d t}\right|_{t=0} d \lambda \mid \leq \frac{M\|P\|_{S}}{A} \leq K\|P\|_{S} .
$$

Now we consider the case that $p \in V \cap \Omega$. Choose $B$ such that $\bar{B} \subset V \cap \Omega$. Let $z \in V \cap \bar{B}$. Take $w, w_{k} \in \partial \Omega$ corresponding to $z$. One can make the appropriate estimate on $\gamma_{z}^{1}$ as above. Let $e$ be a complex unit tangent vector to $\partial \Omega$ at $w$. We construct $S$ such that for all $s \in\left[1-\frac{1}{2} \sigma, 1\right] S$ contains the circles in the complex line through $z+(1-s) \pi_{w}\left(n_{w_{k}}\right)$ and $z+(1-s) \pi_{w}\left(n_{w_{k}}\right)+e$ with center $z+(1-s) \pi_{w}\left(n_{w_{k}}\right)$ and radius $s^{1 /(1+\epsilon / 2)}$. Then

$$
\begin{aligned}
& \left.\left|\frac{d P(\gamma(s) z+t e)}{d t}\right|_{t=0}|=| \frac{d P\left(z+(1-s) \pi_{w}\left(n_{w_{k}}\right)+t e\right)}{d t}\right|_{t=0} \mid \\
& =\left|\frac{1}{2 \pi i} \int_{C\left(0, s^{1 /(1+\epsilon / 2)}\right)} \frac{P\left(z+(1-s) \pi_{w}\left(n_{w_{k}}\right)+t e\right)}{t^{2}} d t\right| \leq \frac{\|P\|_{S}}{s^{1 /(1+\epsilon / 2)}}
\end{aligned}
$$

where $C\left(0, s^{1 /(1+\epsilon / 2)}\right)$ is the circle with center 0 and radius $s^{1 /(1+\epsilon / 2)}$. Hence

$$
\left|\int_{\gamma_{z}^{2}} \frac{d P(\lambda z+t e)}{d t}\right|_{t=0} d \lambda \mid \leq \int_{0}^{1} \frac{M\|P\|_{S}}{s^{1 /(1+\epsilon / 2)}} d s \leq K\|P\|_{S} .
$$

We can choose $S$ to be compact in $\Omega$.

For $z \in V_{k}$ the corresponding $w$ depends continuously on $z$, hence we can choose linearly independent complex unit tangent vectors $e^{1}(z), \ldots, e^{n-1}(z)$ to $\partial \Omega$ at $w$ that depend continuously on $z$. As a consequence of the theorem of Cauchy and the chain rule, we have :

$$
\begin{gathered}
\left.\int_{\gamma_{z}} \frac{d P\left(\lambda z+t e^{j}(z)\right)}{d t}\right|_{t=0} d \lambda=\left.\int_{0}^{1} \frac{d P\left(\lambda z+t e^{j}(z)\right)}{d t}\right|_{t=0} d \lambda \\
=\sum_{i=1}^{n} e_{i}^{j}(z) \int_{0}^{1} D_{i} P(\lambda z) d \lambda=\sum_{i=1}^{n} e_{i}^{j}(z) T_{i}(P)(z), \quad 1 \leq j \leq n-1 .
\end{gathered}
$$

Previously, we already noted that $\sum_{i=1}^{n} z_{i} T_{i}(P) z=P(z)$. Thus, the known numbers $T_{i}(P)(z)$ are solution of the following system of $n$ equations :

$$
\left(\begin{array}{ccc}
e_{1}^{1}(z) & \ldots & e_{n}^{1}(z) \\
\vdots & \vdots & \vdots \\
e_{1}^{n-1}(z) & \ldots & e_{n}^{n-1}(z) \\
z_{1} & \ldots & z_{n}
\end{array}\right)\left(\begin{array}{c}
T_{1}(P)(z) \\
\vdots \\
T_{n-1}(P)(z) \\
T_{n}(P)(z)
\end{array}\right)=\left(\begin{array}{c}
\left.\int_{\gamma_{z}} \frac{d P\left(\lambda z+t e^{1}(z)\right)}{d t}\right|_{t=0} d \lambda \\
\vdots \\
\left.\int_{\gamma_{z}} \frac{d P\left(\lambda z+t e^{n-1}(z)\right)}{d t}\right|_{t=0} d \lambda \\
P(z)
\end{array}\right)
$$

The determinant $\Delta(z)$ of the matrix to the left also exists for $z \in \overline{V_{k}}$, and it depends continuously on $z$. It is nowhere zero, and $\overline{V_{k}}$ is compact, hence its norm is bounded from below. The vectors $z, e^{j}(z) \quad(1 \leq j \leq n-1)$ are linearly independent, as any complex line passing through $\Omega$ intersects $\partial \Omega$ transversally. Hence we can use Cramer's rule to express $T_{i}(P)(z)$ in terms of $\Delta(z)^{-1}, e_{l}^{k}(z), z, P(z)$ and the 
integrals $\left.\int_{\gamma_{z}} \frac{d P\left(\lambda z+t e^{j}(z)\right)}{d t}\right|_{t=0} d \lambda$. Each of those terms can be estimated from above with $C\|P\|_{S}$, hence $T_{i}(P)(z) \leq K_{k}\|P\|_{S}$. Since there is only a finite number of $V_{j}$ 's, we have that $\left\|T_{i}(P)\right\|_{B} \leq K\|P\|_{S}$.

\section{EXTENDING THE OPERATORS $T_{i}$}

Let $f \in H^{\infty}(\Omega)$ such that $f(0)=0$. Let $p, B, S$ be as above. Since $\Omega$ is a Runge domain, there is a sequence $P_{1}, P_{2}, \ldots$ of polynomials that all vanish at 0 , such that $P_{n}$ converges uniformly to $f$ on $S$. Then $T_{i}(f)(z):=\lim _{n \rightarrow \infty} T_{i}\left(P_{n}\right)(z)$ exists for all $z \in B$.

Theorem 4. The function $T_{i}(f)$ is properly defined on $\Omega$. Furthermore, it is in $H^{\infty}(\Omega)$, and $f(z)=\sum_{i=1}^{n} z_{i} T_{i}(f)(z)$.

Proof. It is easy to see that the function $T_{i}(f)$ is properly defined : let $P_{n}, R_{n} \rightarrow f$ uniformly on $S, T$ respectively. Then $\left\|T_{i}\left(P_{n}\right)-T_{i}\left(R_{n}\right)\right\|_{B} \leq K\left\|P_{n}-R_{n}\right\|_{S \cap T} \rightarrow 0$. $\left\|T_{i}(f)\right\|_{B} \leq K\|f\|_{S}$, hence $\left\|T_{i}(f)\right\|_{\Omega} \leq K\|f\|_{\Omega}$. Thus $T_{i}(f) \in L^{\infty}(\Omega)$. Since the sequence of polynomials $T_{i}\left(P_{n}\right)$ converges uniformly on $S$, their limit $T_{i}(f)$ is in $H(B)$. We also have that

$$
\sum_{i=1}^{n} z_{i} T_{i}(f)(z)=\sum_{i=1}^{n} z_{i} \lim _{n \rightarrow \infty} T_{i}\left(P_{n}\right)(z)=\lim _{n \rightarrow \infty} P_{n}(z)=f(z) .
$$

for $z \in B$. As we can repeat this argument for every point $p \in \Omega$ with corresponding neighborhood $B$, the proof is complete.

Lemma 5. Let $f \in A(\Omega)$. For $z \in V_{k}$ with corresponding $w, w_{k} \in \partial \Omega$, let $e(z)$ be a complex unit tangent vector to $\partial \Omega$ at $w$ that varies continuously with $z$. Then

$$
I(z):=\left.\int_{\gamma_{z}} \frac{d f(\lambda z+t e(z))}{d t}\right|_{t=0} d \lambda \quad \in C\left(V_{k}\right) .
$$

Proof. This a fairly standard application of the dominated convergence theorem of Lebesgue. In detail : let $z \in V_{k}$, let $V_{k} \ni z_{n} \rightarrow z$. Then $\gamma_{z_{n}}^{\prime}(s) \rightarrow \gamma_{z}^{\prime}(s)$ for all $s \in[0,1]$. Define $h_{n}$ and $h$ in the following way :

$$
\begin{aligned}
h_{n}(\zeta, s) & := \begin{cases}\frac{f\left(\gamma_{z_{n}}(s) z_{n}+\zeta e\left(z_{n}\right)\right)}{\zeta^{2}} \gamma_{z_{n}}^{\prime}(s), & \text { if } s \in\left[0,1-\frac{1}{2} \sigma\right] ; \\
\frac{f\left(\gamma_{z_{n}}(s) z_{n}+(1-s)^{1+\epsilon / 2} \zeta e\left(z_{n}\right)\right)}{(1-s)^{1+\epsilon / 2} \zeta^{2}} \gamma_{z_{n}}^{\prime}(s), & \text { if } s \in\left[1-\frac{1}{2} \sigma, 1\right] ;\end{cases} \\
h(\zeta, s) & := \begin{cases}\frac{f\left(\gamma_{z}(s) z+\zeta e(z)\right)}{\zeta^{2}} \gamma_{z}^{\prime}(s), & \text { if } s \in\left[0,1-\frac{1}{2} \sigma\right] ; \\
\frac{\left.f\left(\gamma_{z}(s) z+(1-s)\right)^{1+\epsilon / 2} \zeta e(z)\right)}{(1-s)^{1+\epsilon / 2} \zeta^{2}} \gamma_{z}^{\prime}(s), & \text { if } s \in\left[1-\frac{1}{2} \sigma, 1\right] .\end{cases}
\end{aligned}
$$

Then

$$
\begin{aligned}
I\left(z_{n}\right) & =\left.\int_{\gamma_{z_{n}}} \frac{d f\left(\lambda z_{n}+t e\left(z_{n}\right)\right)}{d t}\right|_{t=0} d \lambda \\
& =\int_{0}^{1-\frac{1}{2} \sigma} \int_{C(0, A)} h_{n}(\zeta, s) d \zeta d s+\int_{1-\frac{1}{2} \sigma}^{1} \int_{C(0,1)} h_{n}(\zeta, s) d \zeta d s .
\end{aligned}
$$

For fixed $\zeta$ and $s, h_{n}(\zeta, s)$ converges to $h(\zeta, s)$. Furthermore, for all $n \in \mathbb{N}$ and $s \in\left[1-\frac{1}{2} \sigma, 1\right]$ one has that $\left|h_{n}(\zeta, s)\right| \leq M\|f\|(1-s)^{-1 /(1+\epsilon / 2)}$. For $s \in\left[0,1-\frac{1}{2} \sigma\right]$ 
there is a similar estimate on $h_{n}$. The function $(1-s)^{-1 /(1+\epsilon / 2)}$ is integrable on $[0,1]$. Applying Lebesgue's theorem yields that

$$
I\left(z_{n}\right)=\iint h_{n} \rightarrow \iint h=I(z),
$$

thus $I \in C\left(V_{k}\right)$.

Now let $f \in A(\Omega), f(0)=0$. On $\Omega$ we define $T_{i}(f)(z)$ as above. We now proceed to define $T_{i}(f)$ on $\partial \Omega$. To every $z \in V_{k}$ there correspond $w, w_{k} \in \partial \Omega$. We choose linearly independent complex unit tangent vectors $e^{1}(z), \ldots, e^{n-1}(z)$ to $\partial \Omega$ at $w$ such that they depend continuously on $z \in V_{k}$. Consider the following system of $n$ equations $S Y(f)$ :

$$
\left(\begin{array}{ccc}
e_{1}^{1}(z) & \ldots & e_{n}^{1}(z) \\
\vdots & \vdots & \vdots \\
e_{1}^{n-1}(z) & \ldots & e_{n}^{n-1}(z) \\
z_{1} & \ldots & z_{n}
\end{array}\right)\left(\begin{array}{c}
x_{1} \\
\vdots \\
x_{n-1} \\
x_{n}
\end{array}\right)=\left(\begin{array}{c}
\left.\int_{\gamma_{z}} \frac{d f\left(\lambda z+t e^{1}(z)\right)}{d t}\right|_{t=0} d \lambda \\
\vdots \\
\left.\int_{\gamma_{z}} \frac{d f\left(\lambda z+t e^{n-1}(z)\right)}{d t}\right|_{t=0} d \lambda \\
f(z)
\end{array}\right)
$$

The vectors $z, e^{j}(z) \quad(1 \leq j \leq n-1)$ are linearly independent, since any complex line passing through $\Omega$ intersects $\partial \Omega$ transversally. Thus the system has a unique solution.

Lemma 6. Let $f \in A(\Omega)$ such that $f(0)=0$. Let $z \in V_{k}^{\circ}$. Then $x_{i}=T_{i}(f)(z)$.

Proof. Choose a compact set $S \subset \Omega$ that contains $z$, as in the proof of lemma 2 . Choose a compact set $T \subset \Omega$ such that $S \subset T^{\circ}$. Let $P_{1}, P_{2}, \ldots$ be a sequence of polynomials (all vanishing at 0 ) that converges uniformly to $f$ on $T$. Then $D_{i} P_{n} \rightarrow D_{i} f$ uniformly on $S$, hence, because of the chainrule,

$$
\left.\lim _{n \rightarrow \infty} \int_{\gamma_{z}} \frac{d P_{n}(\lambda z+t e)}{d t}\right|_{t=0} d \lambda=\left.\int_{\gamma_{z}} \frac{d f(\lambda z+t e)}{d t}\right|_{t=0} d \lambda
$$

In the proof of lemma 2 we saw that for a polynomial $P$, the solution $\left(x_{1}, \ldots, x_{n}\right)$ of the system $S Y(P)$ is indeed $\left(T_{1}(P)(z), \ldots, T_{n}(P)(z)\right)$. Taking the limit on both sides of the system of equations $S Y\left(P_{n}\right)$ yields that $x_{i}=T_{i}(f)(z)$.

For $z \in \partial \Omega$ we define $T_{i}(f)(z):=x_{i}$.

Lemma 7. Let $f$ be a function in $A(\Omega)$ that vanishes at 0 . Then $T_{i}(f) \in A(\Omega)$.

Proof. Let $\Delta(z)$ be the determinant of the matrix to the left in $S Y(f)$. We again use Cramer's rule to express $T_{i}(f)(z)$ in terms of $\Delta(z)^{-1}, e_{l}^{k}(z), z, f(z)$ and the integrals $\left.\int_{\gamma_{z}} \frac{d f\left(\lambda z+t e^{j}(z)\right)}{d t}\right|_{t=0} d \lambda$. These are all continuous functions of $z$ on $V_{k}$. Therefore $T_{i}(f)$ is in $C\left(V_{k}\right)$, and repeating this argument for all $k$ yields that $T_{i}(f) \in C(V)$. Hence $T_{i}(f) \in A(\Omega)$.

Theorem 8. Let $\Omega \subset \mathbb{C}^{n}$ be a linearly convex domain with $C^{1+\epsilon}$ - boundary. Then the ideal in $A(\Omega)$ (or $H^{\infty}(\Omega)$ ) consisting of all functions in $A(\Omega)$ (or $H^{\infty}(\Omega)$ ) that vanish at $p \in \Omega$ is generated by the coordinate functions $z_{1}-p_{1}, \ldots, z_{n}-p_{n}$.

Proof. For an $f \in A(\Omega)$ (or $\left.H^{\infty}(\Omega)\right)$, such that $f(0)=0$, we have that

$$
f(z)=\sum_{i=1}^{n}\left(z_{i}-p_{i}\right) T_{i}(f)(z)
$$


and $T_{i}(f) \in A(\Omega)\left(\right.$ or $\left.H^{\infty}(\Omega)\right)$.

\section{Final Remarks.}

In [9] Grangé gave the following example of a convex domain in $\mathbb{C}^{2}$ for which $T_{2}(f)$ is unbounded for a certain $f \in H^{\infty}(\Omega)$ : let $h(x):=\frac{-x}{\log x}$ for $x>0, h(0):=0$. Let

$$
\Omega:=\left\{\left(z_{1}, z_{2}\right) \in \mathbb{C}^{2}:\left|z_{2}\right|<1,\left|z_{1}\right|^{2}+h\left(\left|z_{2}\right|\right)-1<0\right\} .
$$

This shows that the functions $T_{i}(f)$ may fail to solve the Gleason problem on $\mathbb{C}$-convex domains with $C^{1}$-boundary. However, it is possible to solve the Gleason problem for $H^{\infty}(\Omega)$ and $A(\Omega)$ by using different techniques, as the present authors showed in 12 .

A glance at the previous proofs may suggest that our results can be obtained under the weaker assumptions of the next lemma. This lemma however shows that these assumptions are not really weaker at all.

Lemma 9. Let $\Omega$ be a bounded domain with $C^{1}$-boundary such that every complex line passing through $\Omega$ intersects $\partial \Omega$ transversally. Suppose that $\Omega$ intersected with any complex line is connected. Then $\Omega$ is $\mathbb{C}$-convex.

Proof. From the conditions it follows immediately that $\Omega$ is connected. Suppose $\Omega$ is not $\mathbb{C}$-convex. Then it is not weakly linearly convex either, meaning there is a point $z \in \partial \Omega$ such that every complex hyperplane $H$ through $z$ intersects $\Omega$. We take for $H$ the complex tangent space to $\partial \Omega$ at $z$. It contains a complex line that is tangential to $\partial \Omega$ at $z$ and intersects $\Omega$. This contradicts our assumption that such a line intersects $\partial \Omega$ transversally.

\section{REFERENCES}

[1] Andersson, M., Passare, M. and R. Sigurdsson, Complex convexity and analytic functionals I, Report RH-06-95, Science Institute, University of Iceland, 1995.

[2] Andersson, M., Passare, M. and R. Sigurdsson, Complex convexity and analytic functionals II, Report RH-20-2000, Science Institute, University of Iceland, 2000.

[3] Backlund, U. and A. Fällström, On Gleason's problem for $H^{\infty}$, Research Report no.6 (1992), Department of Mathematics, University of Umeå, Sweden.

[4] Backlund, U. and A. Fällström, The Gleason problem for $A(\Omega)$, New Zealand J. Math. 24 (1995), no.1, 17-22.

[5] Backlund, U. and A. Fällström, Counterexamples to the Gleason problem, Ann. Scuola Norm. Sup. Pisa Cl. Sci. (4) 26 (1998), no.3, 595-603.

[6] Backlund, U. and A. Fällström, The Gleason property for Reinhardt Domains, Math. Ann. 308 (1997), 85-91.

[7] Fornæss, J. E. and N. Øvrelid, Finitely generated ideals in $A(\Omega)$, Ann. Inst. Fourier (Grenoble) 33 no. 2 (1983), 77-85.

[8] Gleason, A. M., Finitely generated ideals in Banach algebras, J. Math. Mech. 13 (1964), $125-132$.

[9] Grangé, M., Diviseurs de Leibenson et problème de Gleason pour $H^{\infty}(\Omega)$ dans le cas convexe, Bull. Soc. Math. France 114 (1986), 224-245.

[10] Hörmander, L., Notions of convexity, Birkhäuser Boston, Inc., Boston, MA, 1994.

[11] Khenkin, G. M., Approksimatsiya funktsij v psevdovypuklych oblastyach $i$ teorema Z. L. Leibenzona, Bull. Acad. Polon. Sci. Sér. Sci. Math. Astronom. Phys. 19 (1971), 37-42. (Russian) 
[12] Lemmers, O. and J. Wiegerinck, Reinhardt domains and the Gleason problem, to appear in Ann. Scuola Norm. Sup. Pisa Cl. Sci.

[13] Noell, A., The Gleason problem for domains of finite type, Complex Variables Theory Appl. 4 (1985), 233-241.

[14] Øvrelid, N. Integral representation formulas and $L^{p}$ estimates for the $\bar{\partial}$-equation, Math. Scand. 29 (1971), 137-160.

[15] Øvrelid, N. Generators of the maximal ideals of $A(\bar{D})$, Pacific J. Math. 39 (1971), 219-223.

Department of Mathematics

University of Amsterdam

Plantage Muidergracht 24

1018 TV Amsterdam

The Netherlands

E-mail address, Oscar Lemmers: lemmers@science.uva.nl

E-mail address, Jan Wiegerinck: janwieg@science.uva.nl 